\title{
Effective connectivity during visual processing is affected by emotional state
}

\author{
Miroslaw Wyczesany • Tomasz S. Ligeza • \\ Szczepan J. Grzybowski
}

Published online: 23 October 2014

(C) The Author(s) 2014. This article is published with open access at Springerlink.com

\begin{abstract}
The limitations of our cognitive resources necessitate the selection of relevant information from the incoming visual stream. This selection and prioritizing of stimuli allows the organism to adapt to the current conditions. However, the characteristics of this process vary with time and depend on numerous external and internal factors. The present study was aimed at determining how the emotional state affects effective connectivity between visual, attentional and control brain areas during the perception of affective visual stimuli. The Directed Transfer Function was applied on a 32-electrode EEG recording to quantify the direction and intensity of the information flow during two sessions: positive and negative. These data were correlated with a self-report of the emotional state. We demonstrated that the current mood, as measured by self-report, is a factor which affects the patterns of effective cortical connectivity. An increase in prefrontal top-down control over the visual and attentional areas was revealed in a state of tension. It was accompanied by increased outflow within and from the areas recognized as the ventral attentional network. By contrast, a positive emotional state was associated with heightened flow from the parietal to the occipital area. The functional significance of the revealed effects is discussed.
\end{abstract}

Keywords Visual perception · Attention · Mood - Effective connectivity $\cdot$ Directed transfer function

\author{
M. Wyczesany $(\bowtie) \cdot$ T. S. Ligeza $\cdot$ S. J. Grzybowski \\ Psychophysiology Laboratory, Institute of Psychology, Jagiellonian \\ University, Ingardena 6, PL-30060 Kraków, Poland \\ e-mail: miroslaw.wyczesany@uj.edu.pl \\ T. S. Ligeza \\ e-mail: tsl@poczta.fm \\ S. J. Grzybowski \\ e-mail: sz.grzybowski@uj.edu.pl
}

\section{Introduction}

The processing of emotional stimuli is a complex and nonlinear multi-stage process. On different levels, it is affected by both characteristics of the stimuli itself and numerous cognitive and affective factors such as expectations, cognitive set, previous experience, current mood, or learned strategies of emotional control (Corbetta and Shulman 2002). The influence is especially marked in the case of emotionally valenced content of high biological relevance, which potentially requires a fast and adequate response by the organism. Here we investigate how the current emotional state affects the brain areas involved in the perception of emotional content. We argue that connectivity analysis, which examines the brain in terms of a functional network, will bring us more understanding of the roles played by the areas considered.

It has been proven that stress and anxiety alter the recognition level of negative stimuli as well as the spatial characteristics of visual scene processing. Negative mood has been argued to improve the recognition of threatening content in a state of anxiety. This applies to both expected and unexpected stimuli - irrespective of their location in the visual field (Bishop et al. 2004; Gutiérrez and Gutiérrez Calvo 2011; MacLeod and Hagan 1992; Michalowski et al. 2009). The cost of such alteration in perception characteristics is a simultaneous decrease in efficiency in responding to peripheral, non-emotional stimuli (Rossi and Pourtois 2012). Also, a positive mood is known to affect the spatial scope of the visual field. Most data show a tendency to expand spatial attention (Wadlinger and Isaacowitz 2006). However contrary results can also be found (Vanlessen et al. 2013). The inconsistencies suggest the possible impact of other, uncontrolled factors.

The influence of mood is also observed during the visual processing of symbolic, verbal stimuli so that recognition speed and memory performance can be enhanced when reading emotional words that are congruent with the current 
affective state. Some reports, however, have suggested that this effect remains highly specific and only applies to congruence in discrete emotional states (Flor et al. 1997; Kiefer et al. 2007; Niedenthal and Setterlund, 1997, 1994). Late ERP effects characterized by an increase in the late positivity potential (LPP), or a decrease in the N400 component, in response to positive words may be attributed to the "default" positive mood during typical laboratory conditions and could also reflect the mood congruency effect (positivity offset; Herbert et al. 2006; Herbert et al. 2008; Kiefer et al. 2007). On the other hand, very early effects related to sensory analysis (within $100 \mathrm{~ms}$ of word onset) could point to negativity bias, with higher responses evoked by negative words, but only under more arousing circumstances, i.e. in an emotional context (Grzybowski et al. 2014).

Despite the boosting of initial phases of perceptual processing in a negative state, overall behavioral performance can still deteriorate. The higher cognitive functions, from the late stages of perception up to executive processes, can be altered in negative mood (Li et al. 2013), or even disrupted during serious stress or anxiety, which results in lowered performance of goal-related activity due to possible depletion of available resources (Shackman et al. 2011).

To understand affective modulation of perception, it is important to identify what particular stages of processing are affected, since they collectively make up the observed effects. In terms of attention, selective attenuation or enhancement is often considered as a gating mechanism. However, attention is not a unitary phenomenon and two general systems can be distinguished. The first one, the dorsal attentional network (DAN), focuses on the information that is relevant to the current task or goal-related requirements. It represents topdown control over the perceptual system and is responsible for both spatial and feature-related attention. Anatomically, it is identified as the dorsal fronto-parietal network and is composed of bilateral frontal eye fields (FEF) and intra-parietal sulci with superior parietal lobules (IPS/SPL; Corbetta and Shulman 2002; Serences et al. 2005). This network can exert specific control over the perceptual system, from primary to association visual areas in striate and extrastriate cortices (Ruff et al. 2008). Another system, known as the ventral attentional network (VAN), controls for stimuli-driven shifts of attention. Its main function is reorienting the resources toward behaviorally relevant stimuli even when they are unexpected or unattended. It relies on a distributed network located mostly in the right hemisphere: part of the middle and inferior frontal gyrus (MFG/IFG), anterior insula (aIns) and temporoparietal junction (TPJ). The latter is thought to provide an interface with the dorsal network. The ventral system is also influenced by the subcortical limbic structures (amygdala), which can also contribute to the regulation of perceptual sensitivity (Corbetta et al. 2008; Kotani et al. 2009; Salmi et al. 2009).
The two attentional systems are inseparably bound, while providing prioritized processing of relevant information. Current models assume inhibition of the ventral system by the dorsal during task-engagement, which prevents unnecessary distraction by salient but non-relevant stimulation. On the other hand, the ventral system is important for reorienting the dorsal system when important changes in the environment occur (Frank and Sabatinelli 2012; Todd et al. 2005). Yet because investigations into the precedence of neural activations in both systems have yielded inconsistent results, the details and causality of this process are still not clear (Corbetta et al. 2008; Fox et al. 2006). It has also been proposed that the two systems act together to regulate the range of information entering conscious perception (Asplund et al. 2010).

A number of models have already been proposed to explain the causal relationships within the perceptual-attentional system and their role in mood-related changes in perception. It is possible that increased focus on emotional content during an anxiety state observed especially for negative stimuli is based on sensitization of the amygdala toward potential threat signals. Then, the amygdala could further influence the sensory cortex by increasing its activation directly - by neural reentrant connections - and more generally via the locus coeruleus and norepinephrine neuromodulatory paths (Corbetta et al. 2008; Vuilleumier 2005). An alternative explanation based on the attentional control theory (Eysenck et al. 2007; Fiedler et al. 2003) points out that stress and anxiety facilitate the bottom-up, stimulus-driven system, which may transiently prevail over top-down control. Another possibility is a tonic increase of prefrontal control over the visual areas in a negative emotional state in order to enforce utilization of more resources for identifying potential negative stimuli (Vanlessen et al. 2013). The prefrontal influence is also suggested as a plausible mechanism for moodrelated alterations in cognitive processes, including broadening of attentional scope in a positive emotional state (Rowe et al. 2007; Wadlinger and Isaacowitz 2006). Since these mechanisms can act in parallel, the alternative views need not be contradictory.

The existing models of perceptual control are based to a large extent on fMRI data, characterized by low temporal resolution and thus rather correlational in their nature. To step beyond these limitations, it would be beneficial to utilize methods that can determine the paths of neural information flow and thus allow inferences to be made about the causality of brain processes. In the present article, we investigate moodrelated changes in effective connectivity when attending emotional pictures by means of Directed Transfer Function (DTF) applied on the EEG recording (Blinowska et al. 2004; Korzeniewska et al. 2003). This autoregressive method is based on Granger causality principles and provides an estimation of the amount and direction of information flow based on signals from multiple electrodes while controlling the 
familywise alpha level (Kamiński et al. 2001). The procedure was designed to quasi-simultaneously record brain activity and self-reported affective state while attending emotional pictures. The passive viewing context was chosen to minimize the interference of cognitive activity with the emotional state (Wyczesany et al. 2008). The subjects took part in two separate sessions, positive and negative, with mood induction by means of highly valenced stimuli to make the emotional state more variable across subjects (Westermann et al. 1996; Wyczesany et al. 2009). Self-assessment scores were then correlated with DTF values separately in both sessions. Because the analysis is carried out using the actual state of the subject as the independent variable, a design such as this which includes direct assessment of the current affective state - allows for inference even when the mood induction is not fully successful.

It was hypothesized that a negative emotional state would be related to increased feedback from the prefrontal area to both attentional systems and this effect would be visible especially for negative pictures. This would reflect sensitization to threat-related content. Moreover, increased flow from the ventral to the dorsal attentional network (via the TPJ) was expected as a marker of facilitation of bottom-up processes and simultaneous inhibition of top-down attentional control associated with the increased role of stimuli salience. Finally, it was posited that negative stimuli - biologically relevant as they signal potential danger and thus require immediate responses - would reveal stronger effects than those observed for positive ones.

Additional computations were performed using prestimulus alpha power over the visual cortex to integrate the connectivity analysis with classic EEG methods. Its level during an idle period, just before the presentation of the stimulus, is thought to reflect the active top-down influence that modulates the sensitivity of the perceptual system (Dijk et al. 2008).

\section{Materials and methods}

\section{Procedure}

The procedure was compliant with the directives of the Helsinki Declaration (1975, revised 2000) and approved by the Ethical Committee of the Institute of Psychology, Jagiellonian University. Informed consent was obtained from all subjects. Forty-two undergraduate student volunteers (23 women; mean age 20.1, SD 1.7), participated in the study after signing a written consent form. All of them were medicationfree with no reported history of neurological and psychiatric diseases - or of substance abuse - and had normal or corrected-to-normal vision. During the procedure, the subjects were seated in an air-conditioned soundproof cabin before a 24" LCD monitor.

There were two randomly sequenced sessions, one positive and one negative, which were determined by the valence of the slides displayed. Forty emotional pictures were chosen from the International Affective Picture System (IAPS) database (Lang et al. 1999). Half of them had a high negative rating (mean 2.1) and half had a high positive rating (mean 7.2). Both sets were matched for arousal (mean 5.7 and 5.6 respectively) and additional precautions were taken to equate them - where possiblein terms of content and complexity. Both sets thus included humans (seven photos depicted faces in the positive set and nine in the negative set), animals, landscapes, and food. ${ }^{1}$ Each session started with a presentation of ten pictures randomly chosen from the main set (full-screen, $2 \mathrm{~s}$, ISI $0.5 \mathrm{~s}$ ). They were primarily intended to influence the participants' mood and were not analyzed in the connectivity computations that follow. The main section of the experiment in which the emotional stimuli were presented then began. The pictures were intermixed with adjectives (with a response scale provided) intending to measure the subjects' current emotional state. The pictures and adjectives were presented in random order. Apart from the number of repetitions of each item, which was fixed, no additional constraints were set for the sequence of stimuli; there could be one or more pictures followed by one or more adjectives. The parameters for the picture presentation were as follows: $0.5 \mathrm{~s}$ blank screen, $0.5 \mathrm{~s}$ fixation cross, $2 \mathrm{~s}$ display time, ISI randomly 0.3 to $1 \mathrm{~s}$, full-screen, each picture repeated four times, and 160 trials in total. As for the adjectives, 29 items taken from the Polish version of the UWIST Mood Adjective Checklist (UMACL; Matthews et al. 1990) were used. The adjectives were followed by a questionnaire-based inquiry "Do you feel that way?" - with a four-point rating scale. Each adjective (black letters on a grey background, Liberation Sans font, $24 \mathrm{p}$ ) was repeated three times and preceded by a blank screen interval (random length from 0.3 to $1 \mathrm{~s}$ ) and a fixation cross $(0.5 \mathrm{~s})$. Positive and negative sessions were separated by a break. The procedure was then stopped and the subjects were free to leave the experimental room. This was intended to attenuate the mood induction effect from the previous session. The schema of the experimental procedure is depicted in Fig. 1.

Data recording and analysis

EEG recording was carried out using a Biosemi Active Two device equipped with 32 active electrodes placed on a 10-20 headcap and four additional leads located above and

\footnotetext{
${ }^{1}$ The IAPS picture numbers for the positive set were: $1710,1722,2208$, 2209, 2345, 4599, 4641, 4650, 5270, 5450, 5470, 5623, 7220, 7230, $7270,7502,8300,8420,8496,8500$; and for the negative set: 2710,3015 , $3220,3230,3301,3350,3550,6570,7380,9253,9300,9400,9405$, 9420, 9500, 9520, 9560, 9570, 9571, 9920.
} 
Fig 1 The time-course scheme of the procedure

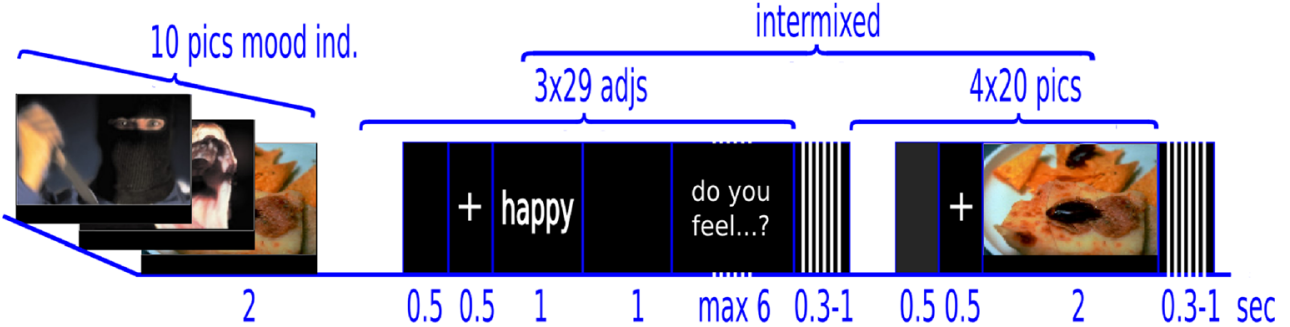

below the right eye and in the external canthi of both eyes. The preprocessing was done using an EEG lab toolbox (Delorme et al. 2011), where the signal was offline referenced to linked mastoids (recorded with two more electrodes) and filtered in a range of $1 \mathrm{~Hz}$ to $45 \mathrm{~Hz}$ with zero phase-shift filters. Two-second epochs (0 to $2 \mathrm{~s}$ relative to picture onset) were extracted. No oculomotor correction was applied in the case of DTF analysis; instead, segments including artifacts were rejected. First, to detect eye blinks, a $100 \mu \mathrm{V}$ rejection threshold was set for all of the electrodes, including the one located over the right eye, where the blinks were most prominent. Next, to reveal significant eye movement artifacts (especially saccades), Independent Component Analysis was used to separate horizontal movement signals, which were identified using the criteria of scalp distribution and temporal characteristics (Jung et al. 2000). Trials in which the amplitude of the eye movement component exceeded $30 \mu \mathrm{V}$ were rejected. Based on the Akaike information criterion (AIC), the autoregression model order for analysis was set to 7. A check was made before the connectivity analysis to see if the length of data remaining after the rejection procedure was sufficient for DTF calculations. The following formula was applied: W $\geq 10 *(\mathrm{pM} / \mathrm{N})$ where: $\mathrm{W}$ - required minimum window length in samples, $\mathrm{p}$ - model order, $\mathrm{M}$ - number of channels and $\mathrm{N}$ - total number of epochs for all trials within each valence condition (Korzeniewska et al. 2003). The criterion was fulfilled for all subjects. DTF calculations were made using Multar software (Department of Biomedical Physics, University of Warsaw; www.eeg.pl). Based on EEG montage brain atlases (Kaiser 2007; Okamoto et al. 2004), the electrodes corresponding to the following regions of interest (ROIs) were selected for connectivity analysis: right inferior frontal gyrus, IFG (VAN): F8; right temporoparietal junction, RTPJ (VAN): CP6; left/right intra-parietal sulci, IPS (DAN): CP1, P3, CP2, P4; striate/extrastriate visual cortex: O1, $\mathrm{Oz}, \mathrm{O} 2, \mathrm{PO} 3, \mathrm{PO} 4$; left/right prefrontal cortex; and PFC: Fp1, AF3, AF4, Fp2. Since the DTF method estimates the information flow based on the phase difference between channels, it remains insensitive to the problem of volume conduction, as the electromagnetic fields propagate nearinstantaneously with no phase differences between electrodes (Kamiński and Blinowska 2014). This allows for more precise location of underlying structures, with no additional signal processing (such as Laplace or beamforming; Nunez et al. 1997). Normalized DTF values for all pairs of electrodes between the ROIs were then calculated for the frequency important in attentional processes (beta $14 \mathrm{~Hz}-25 \mathrm{~Hz}$ ), which is especially significant in the visual domain (Gola et al. 2013; Kamiński et al. 2012; Kus et al. 2008).

To control the quality of autoregressive model fitting, the residual noise matrices were determined for all subjects. The distributions of the DTF values obtained were checked to identify and reject possible extremes, which were defined as falling below Q1-1.5*IQR or above Q3+1.5*IQR (Q - quartile; IQR - interquartile range; Tukey 1976; Wyczesany et al. 2014).

To determine the valence of the subjects' mood, the selfreport scores were calculated separately for both sessions according to the original UMACL instructions. This yielded three dimensions: Energetic Arousal (EA: a measure of the organism's overall arousal level), Tension Arousal (TA: a negatively valenced state of tension, worry, and anxiety) and Hedonic Tone (HT: a pleasant and enjoyable state).

Next - after ensuring normal distributions of data by means of a series of Kolmogorov-Smirnov tests - the relationships between the subjective scales and DTF values were estimated separately in positive and negative sessions using Pearson's $r$ correlations. Hence, flow increases or decreases refer to positive or negative correlations respectively, with the subjective scales given observed for the extracted epochs. Finally, because there were multiple comparisons involved, the False Discovery Rate (FDR) procedure (Benjamini and Hochberg 1995) was applied for all flows between the ROIs considered to correct significance levels.

The one-second epochs directly preceding the pictures were extracted for prestimulus spectral analysis. They were corrected for ocular artifact by subtracting ICA components classified as eye-movements and blinks based on their spatial and temporal characteristics (Jung et al. 2000). The spectral power for the alpha band $(8 \mathrm{~Hz}-12 \mathrm{~Hz})$ was calculated using the multitaper method (three orthogonal Slepian tapers) on the electrodes over the visual cortex ROI. Spearman correlations between alpha power and DTF values, measuring top-down information transfer 
from the left and right prefrontal cortex (Fp1, AF3 / $\mathrm{Fp} 2, \mathrm{AF} 4)$ to the visual area (PO3, O1, Oz, O2, PO4), were calculated separately for the negative and positive sessions.

\section{Results}

The sessions differed significantly in terms of all three subjective dimensions, which were assessed using repeated measures t-tests. The direction of changes confirmed the efficiency of the mood induction, which affected the entire duration of each session. When compared to the positive pictures, the negative ones were associated with higher tension (TA) and lower energetic arousal (EA), as well as with more negative valence (lower HT scores; see Table 1).

The autoregression model fitting for the EEG signal was good enough in all subjects, i.e. mean RV values did not exceed $13 \%$. The most significant correlations between subjective scales and connectivity measures were found for the TA scale; in all cases the affected sources increased their outflow with elevated tension. In the negative session, the following directions of flow showed significant effects: from Fp1 to CP1, CP2, P4 and CP6; from AF3 to CP1, CP2, P3, P4, CP6, PO3 and PO4; and from F8 to Fp1, AF3 and CP6 electrodes. In the positive session, the number of significantly affected flows was lower: from AF3 to CP1 and P3, and from F8 to Fp1, Fp2, AF3 and CP6 electrodes. The effects of the positive valence (higher HT scores) were seen as increased flow from the CP6 to CP2 electrode for both sessions, and from the $\mathrm{CP} 2$ to the $\mathrm{O} 1$ electrode only in the negative session. Detailed correlation coefficients with corresponding $\mathrm{p}$-values (both raw and FDR-adjusted) are shown in Tables 2 and 3, and are visualized on the scalp surface in Fig. 2. For further interpretation of the results, the significant effects were mapped onto the putative underlying brain structures using the predefined ROIs (see Fig. 3 for the negative session). In terms of ROIs, the observed effects of the TA scale included the following flows: In the negative session - from the left PFC to the bilateral IPS and visual area, and from the right IFG to the left PFC and the right TPJ; In the positive session from the left PFC to the left IPS, and from the right IFG to the prefrontal area as well as to the right TPJ. The effects of the HT scale included increased flow from the right TPJ to the right IPS in both sessions, as well as from the right IPS to the visual area in the negative session.

Significant negative correlation between the alpha power and average DTF from the left prefrontal cortex to the visual area was observed in the negative session (Spearman's rho= $-0.45, p=0.005$ ), while the positive one revealed no significant relationships.

\section{Discussion}

We investigated the mood-related changes of connectivity between the brain's perceptual, attentional, and prefrontal control centres. We assumed that their effective connectivity could underlie the dynamic modulation of perception and the processing of emotional stimuli, which are associated with the current affective state. The results show that the level of Tension Arousal (TA), as a scale strongly linked to negative valence, turned out to be the most influential factor affecting the strength of connectivity. Some results for Hedonic Tone (HT), which, on the other hand, is strongly linked to positive valence, were also observed. In contrast, Energetic Arousal (EA) did not yield any significant results. Since the EA scale is associated mostly with the energetic and not the valence aspect of the emotional state (Wyczesany et al. 2010), the null result suggests that the valence may be the main factor influencing information flow, which is discussed further elsewhere in the text.

As expected, mood-related changes in effective connectivity were more prominent in the negative session. This is not surprising, since swift and adequate responses to external signals are more critical for the organism in conditions that carry a potential threat. This confirms other reports that have shown heightened coupling of brain regions (Moratti et al. 2011), or increases in brain activations, during perceptual tasks observed in a state of tension when compared to those observed in relaxed and safe conditions (Dalton et al. 2005). Although the effects seen in the positive session were usually weaker, it is worth noting that many of them were apparently a subset of those visible during the negative session. This supports the claim that the relationships observed between the current emotional state and the effective connectivity can be considered as a more general pattern related to emotional arousal, which are therefore not limited to specific experimental conditions.

Table 1 Group means of subjective scores with differences between sessions

\begin{tabular}{|c|c|c|c|c|c|c|}
\hline & negative session mean & positive session mean & change (NEG-POS) & $\mathrm{t}$ & df & significance $(\mathrm{p})$ \\
\hline Energetic Arousal (EA) & 24.13 & 25.18 & -1.05 & -2.15 & 41 & 0.038 \\
\hline Tension Arousal (TA) & 19.86 & 15.01 & 4.85 & 6.93 & 41 & $<0.001$ \\
\hline Hedonic Tone (HT) & 22.79 & 29.93 & -7.14 & -8.75 & 41 & $<0.001$ \\
\hline
\end{tabular}


Table 2 Significant correlations between DTF values and Tension Arousal scores observed in the $\beta$ frequency band

Tension Arousal effects

\begin{tabular}{|c|c|c|c|c|c|c|}
\hline \multirow[b]{2}{*}{ flow direction } & \multicolumn{3}{|c|}{ negative pictures } & \multicolumn{3}{|l|}{ positive pictures } \\
\hline & correlation (r) & raw significance $(p)$ & $\begin{array}{l}\text { FDR-corrected } \\
\text { significance }(p)\end{array}$ & correlation (r) & Raw significance (p) & $\begin{array}{l}\text { FDR-corrected } \\
\text { significance }(p)\end{array}$ \\
\hline \multicolumn{7}{|l|}{$\mathrm{LPFC} \rightarrow \mathrm{IPS}$} \\
\hline $\mathrm{AF} 3 \rightarrow \mathrm{CP} 1$ & 0.31 & 0.02 & 0.03 & 0.43 & 0.00 & 0.02 \\
\hline $\mathrm{AF} 3 \rightarrow \mathrm{CP} 2$ & 0.46 & 0.00 & 0.01 & - & & \\
\hline $\mathrm{AF} 3 \rightarrow \mathrm{P} 3$ & 0.27 & 0.04 & 0.05 & 0.25 & 0.05 & ns \\
\hline $\mathrm{AF} 3 \rightarrow \mathrm{P} 4$ & 0.33 & 0.02 & 0.03 & - & & \\
\hline $\mathrm{Fp} 1 \rightarrow \mathrm{CP} 1$ & 0.31 & 0.03 & 0.03 & - & & \\
\hline $\mathrm{Fp} 1 \rightarrow \mathrm{CP} 2$ & 0.37 & 0.01 & 0.03 & - & & \\
\hline $\mathrm{Fp} 1 \rightarrow \mathrm{P} 3$ & - & & & - & & \\
\hline $\mathrm{Fp} 1 \rightarrow \mathrm{P} 4$ & 0.34 & 0.02 & 0.03 & - & & \\
\hline \multicolumn{7}{|l|}{$\mathrm{RIFG} \rightarrow \mathrm{PFC}$} \\
\hline $\mathrm{F} 8 \rightarrow \mathrm{AF} 3$ & 0.29 & 0.03 & 0.05 & 0.28 & 0.04 & 0.05 \\
\hline $\mathrm{F} 8 \rightarrow \mathrm{Fp} 1$ & 0.31 & 0.03 & 0.05 & 0.34 & 0.01 & 0.03 \\
\hline $\mathrm{F} 8 \rightarrow \mathrm{AF} 4$ & - & & & - & & \\
\hline $\mathrm{F} 8 \rightarrow \mathrm{Fp} 2$ & - & & & 0.33 & 0.02 & 0.03 \\
\hline \multicolumn{7}{|l|}{$\mathrm{LPFC} \rightarrow \mathrm{RTPJ}$} \\
\hline $\mathrm{AF} 3 \rightarrow \mathrm{CP} 6$ & 0.36 & 0.01 & 0.03 & - & & \\
\hline $\mathrm{Fp} 1 \rightarrow \mathrm{CP} 6$ & 0.33 & 0.02 & 0.03 & - & & \\
\hline $\mathrm{AF} 4 \rightarrow \mathrm{CP} 6$ & - & & & - & & \\
\hline $\mathrm{Fp} 1 \rightarrow \mathrm{CP} 6$ & - & & & - & & \\
\hline \multicolumn{7}{|l|}{$\mathrm{LPFC} \rightarrow \mathrm{Occ}$} \\
\hline $\mathrm{AF} 3 \rightarrow \mathrm{PO} 4$ & 0.46 & 0.00 & 0.02 & - & & \\
\hline $\mathrm{AF} 3 \rightarrow \mathrm{PO} 3$ & 0.39 & 0.01 & 0.03 & - & & \\
\hline $\mathrm{AF} 3 \rightarrow \mathrm{O} 1$ & - & & & - & & \\
\hline $\mathrm{AF} 3 \rightarrow \mathrm{O} 2$ & - & & & - & & \\
\hline $\mathrm{AF} 3 \rightarrow \mathrm{Oz}$ & - & & & - & & \\
\hline $\mathrm{Fp} 1 \rightarrow \mathrm{PO} 4$ & - & & & - & & \\
\hline $\mathrm{Fp} 1 \rightarrow \mathrm{PO} 3$ & - & & & - & & \\
\hline $\mathrm{Fp} 1 \rightarrow \mathrm{O} 1$ & - & & & - & & \\
\hline $\mathrm{Fp} 1 \rightarrow \mathrm{O} 2$ & - & & & - & & \\
\hline $\mathrm{Fp} 1 \rightarrow \mathrm{Oz}$ & - & & & - & & \\
\hline \multicolumn{7}{|l|}{$\mathrm{RIFG} \rightarrow \mathrm{RTPJ}$} \\
\hline $\mathrm{F} 8 \rightarrow \mathrm{CP} 6$ & 0.38 & 0.01 & 0.01 & 0.28 & 0.04 & 0.04 \\
\hline $\mathrm{P} 4 \rightarrow \mathrm{O} 1$ & - & & & - & & \\
\hline $\mathrm{P} 4 \rightarrow \mathrm{Oz}$ & - & & & - & & \\
\hline $\mathrm{P} 4 \rightarrow \mathrm{O} 2$ & - & & & - & & \\
\hline
\end{tabular}

A massive intensification of information flow from the left prefrontal cortex, related to emotional tension, confirmed our hypotheses. The affected areas included those putatively corresponding both to attentional systems (DAS: both IPS under the $\mathrm{CP} 1, \mathrm{P} 3, \mathrm{CP} 2, \mathrm{P} 4$ electrodes; VAS: right TPJ under the CP6 electrode) as well as to the visual cortex $(\mathrm{O} / \mathrm{PO}$ electrodes). This vast increase in signal propagation can be interpreted as a heightened top-down influence enforced on the attentional and perceptual systems. Such a pattern could possibly underlie mood-related sensitization to potential threat signals, which can be referred to as "hyper-vigilance". Indeed, the strengthening of top-down connections is suggested as a phenomenon accompanying the prioritized processing of affective content (Lee et al. 2010).

This also confirms the fMRI connectivity data, which shows attention-dependent changes in coupling between the 
Table 3 Significant correlations between DTF values and Hedonic Tone scores observed in the $\beta$ frequency band

Hedonic Tone effects

\begin{tabular}{|c|c|c|c|c|c|c|}
\hline \multirow[b]{2}{*}{ flow direction } & \multicolumn{3}{|c|}{ negative pictures } & \multicolumn{3}{|c|}{ positive pictures } \\
\hline & correlation (r) & raw significance $(p)$ & $\begin{array}{l}\text { FDR-corrected } \\
\text { significance }(\mathrm{p})\end{array}$ & correlation (r) & raw significance $(\mathrm{p})$ & $\begin{array}{l}\text { FDR-corrected } \\
\text { significance }(p)\end{array}$ \\
\hline \multicolumn{7}{|l|}{$\mathrm{RTPJ} \rightarrow \mathrm{IPS}$} \\
\hline $\mathrm{CP} 6 \rightarrow \mathrm{CP} 1$ & - & & & - & & \\
\hline $\mathrm{CP} 6 \rightarrow \mathrm{P} 3$ & - & & & - & & \\
\hline $\mathrm{CP} 6 \rightarrow \mathrm{CP} 2$ & 0.34 & 0.01 & 0.05 & 0.38 & 0.01 & 0.05 \\
\hline $\mathrm{CP} 6 \rightarrow \mathrm{P} 4$ & - & & & - & & \\
\hline \multicolumn{7}{|l|}{$\mathrm{RIPS} \rightarrow \mathrm{Occ}$} \\
\hline $\mathrm{CP} 2 \rightarrow \mathrm{O} 1$ & 0.31 & 0.02 & 0.05 & - & & \\
\hline $\mathrm{CP} 2 \rightarrow \mathrm{Oz}$ & 0.37 & 0.01 & ns & - & & \\
\hline $\mathrm{CP} 2 \rightarrow \mathrm{O} 2$ & - & & & - & & \\
\hline $\mathrm{P} 4 \rightarrow \mathrm{O} 1$ & - & & & - & & \\
\hline $\mathrm{P} 4 \rightarrow \mathrm{Oz}$ & - & & & - & & \\
\hline $\mathrm{P} 4 \rightarrow \mathrm{O} 2$ & - & & & - & & \\
\hline
\end{tabular}

visual cortex and the IPS (Büchel and Friston 1997; McLaughlin et al. 2009). Connectivity between the occipital and prefrontal areas is also observed to increase during involvement in demanding perceptual tasks (Rowe et al. 2005). The causal role of the left dorsolateral PFC in intensifying the processing of negative stimuli has also been
Fig. 2 Directions of information flow (normalized DTF values) which significantly correlated with the self-report scores in the $\beta$ freq band
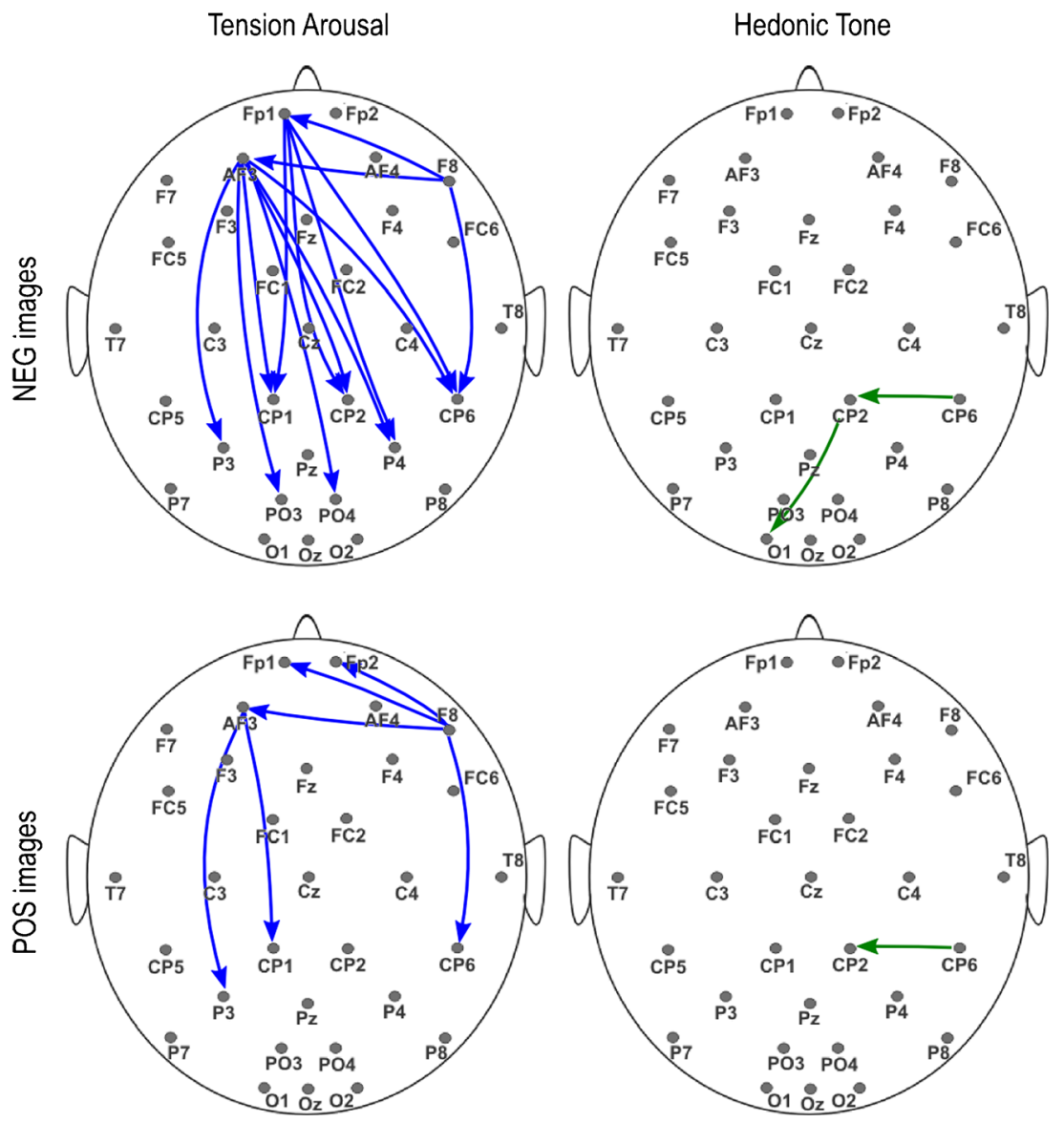


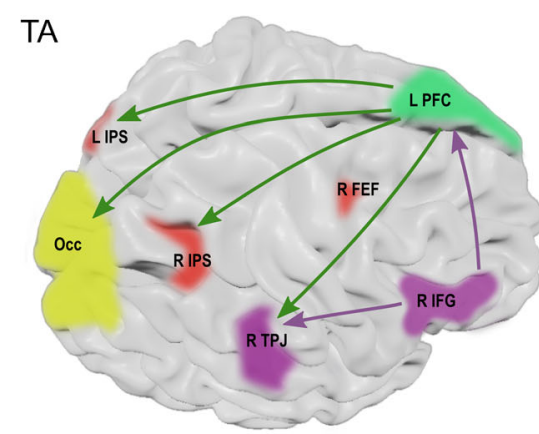

Fig. 3 Projection of observed effects onto the brain surface with location of putative structures involved. Negative session effects are shown. Solid lines denote positive correlations. $L$ PFC left prefrontal cortex, $R I F G$

demonstrated in a TMS study (D'Alfonso et al. 2000), where dorsolateral PFC stimulation resulted in disruption of attention toward negatively valenced faces.

It is also important to note that the connectivity results were supported by the prestimulus alpha power analysis. This activity - recorded over the visual cortex during the period of anticipation of visual stimuli - can be considered a reverse marker of the top-down influence. It was shown to covary with the level of perceptual sensitivity and ability to discriminate visual stimuli and reflect the mechanism of visual input regulation (Dijk et al. 2008; Min and Herrmann 2007; Romei et al. 2010). The strong negative correlation between the prestimulus alpha and the prefrontal-visual connectivity confirms our interpretation relating this top-down link with modulation of the early, perceptual stage of visual processing. It is worth pointing out that this relationship was observed only in the negative session, which shows the importance of this modulatory process in the case of negative, biologically relevant stimuli.

Although we focus here on the automatic boosting of attention, an alternative interpretation cannot be ruled out. As can be seen, the prefrontal effects were limited to the left side. Indeed, some data have shown lateral specialization of the dorsolateral part of the PFC (DLPFC) as a control center. It has been suggested that the left DLPFC plays a dominant role in continuously monitoring the demands of the current situation and imposing automatic control over the attentional systems (MacDonald et al. 2000; Stuss et al. 2001; Vanderhasselt et al. 2009). However, the DLPFC regions are also known to be crucial for affective control (Buhle et al. 2013). The right DLPFC (compared with the left DLPFC) has been linked more frequently with the regulation of emotional processing in highly demanding conditions, such as in the presence of high intensity stimuli, in which additional cognitive control resources are required (Ochsner et al. 2012; Silvers et al. 2014). The lack of right DLPFC effects (compared with the left DLPFC) may therefore be explained by the passive procedure, where subjects were not engaged in any explicit, demanding task. Conversely, the activity in the left DLPFC

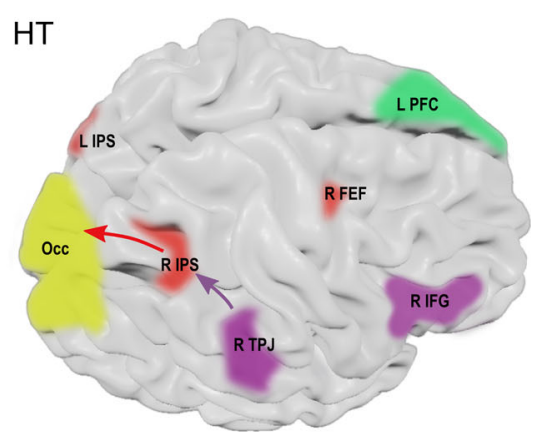

right inferior frontal gyrus, $R F E F$ right frontal eye field, TPJ temporoparietal junction, IPS intraparietal sulcus, Occ occipital visual cortex

could be related to an implicit form of affect regulation. Such a process could be initiated by the simple registration of sensory inputs and could take place without awareness and downregulate the negative affect (Gyurak et al. 2011). There are data showing that the state of anxiety (which is closely related to tension arousal in our study) lowers the threshold for evoking a fear response to threatening stimuli which, on the other hand, can initiate affective control mechanisms (Bishop 2008). Strong emotional tension (especially in the negative session) could provoke mechanisms for mood modulation to some extent and thus additionally augment implicit downregulation of affect (Tupak et al. 2014).

This is not, however, contradictory to our main line of reasoning, which points to modulatory influences on the perceptual/attentional system. The mood-related control of attention and possible down-regulation of affect are known to be partly related to each other (Okon-Singer et al. 2007), and these mechanisms could overlap during the negative session (it is unlikely that the positive session would have elicited significant down-regulation of affect). While moodrelated control of attention could be seen in the positive and negative sessions, down-regulation of affect may represent additional outflows from the PFC regions in the negative session. In summation, these two facts support the importance of automatic attention control, which we propose as underlying our results.

Self-reported tension was also associated with an increased flow from the areas recognized as the ventral attentional network (F8 electrode mapped onto the right IFG) to certain other PFC regions. This was also accompanied by strengthening of connectivity with the right TPJ (located under the CP6 electrode). Importantly, these effects were visible for both sessions, which indicates that they may appear prior to full stimuli evaluation as a tonic sensitization to potential changes in the environment. Indeed, the role of the right IFG in modulating different features of visual attention by influencing the occipital and parietal areas has been widely shown (Corbetta and Shulman 2002; Hedden and Gabrieli 2006; Zanto et al. 2011). In line with these observations, this effect 
can be attributed to the increased involvement of the VAN observed at the connectivity level.

The predicted link between the ventral and taskoriented networks was identified as a specific and spatially limited flow from the CP6 electrode (over the right TPJ) to the CP2 electrode (over the right IPS). Thus the hypothesis that the path of communication between both attentional networks involves the right TPJ as an interface (Serences et al. 2005; Vossel et al. 2013) received some support. However, contrary to this hypothesis, the flow of the link under consideration was found to decrease in a negative emotional state when what was expected was the inhibition of the task-oriented attentional network by the stimulus-driven one. This reversed relationship leaves room only for speculative interpretations. Hence, it is possible that the observed flow represents a facilitatory instead of an inhibitory influence from the ventral network. Such an explanation could be supported by the data showing that behavioral, goal-directed performance related to expectation of visual cues is improved with decreased Granger influence from the right TPJ to the right IPS, which does indeed suggest the inhibitory character of this connection (Wen et al. 2012).

Positive valence of the emotional state (increased HT scores) was found to be associated with a heightened flow from the electrodes over the right IPS to the occipital area. This effect possibly relates to the extension of the spatial scope of attention in a positive mood, which is accompanied by a simultaneous decrease in sensitivity to negative signals. Some data suggest the causal role of the right IPS in regulating this trade-off by strengthening the top-down spatial features of attention (Oliveri et al. 2010). Our data support this view, by showing increased flow from the parietal area to the visual cortex in more positive affect.

\section{Conclusions and limitations}

As can be seen, the DTF method proved to be a valuable tool which allowed us to ascertain the direction and changes of information flow based on EEG data. The data presented shed more light on the interaction between perceptual, attentional, and control brain areas and the emotional state as a factor influencing the process of visual perception. Although both the positive and negative conditions produced similar observations, the latter revealed more prominent effects.

We showed a tension-related increase of information outflow activity located in the left lateral prefrontal area. This effect was interpreted as sensitization driven from the topdown. It was found to involve a simultaneous influence on multiple cortical areas and thus to affect different stages of information processing. It was also supposed that negative conditions recruited some additional mechanisms for implicit, affective down-regulation, which could add to the perceptual/ attentional control activity. In conclusion, our result supports the role of the prefrontal cortex as a crucial area for the integration of cognitive functions and the modulation of the entire attentional/perceptual system. Reported tension was also associated with increased connectivity within and from the areas recognized as the dorsal attentional network (RIFG and RTPJ). This would seem to be a plausible neural mechanism underlying the sensitization of the stimuli-driven attention. On the other hand, positive mood (HT scale) was related to increased flow from the right parietal area to the visual cortex, which could underlie changes in attentional scope.

Most importantly, we managed to demonstrate that the current mood, as measured by self-report, is a factor that affects the patterns of effective cortical connectivity. A multiple-item checklist tool was chosen for use in the study, which was intended for more implicit mood measurement, where the researcher's intentions are not easily disclosed. The intermixing of emotional stimuli with adjectival items could be less susceptible to the influence of demand characteristics, since the consideration of possible experimental expectations in subjects is limited when they are focused on a pending procedure. These factors suggest that the accuracy of such quasi-simultaneous measurement is possible and better than a retrospective check (Hurlburt and Heavey 2001; Thomas and Diener 1990). It is known on the other hand that even simple cognitive tasks, such as rating or labelling emotional stimuli, can reduce the reactivity of limbic structures (Lieberman et al. 2007; Taylor et al. 2003). We argue, however, that because mood assessment was separated from emotional stimuli in our procedure this effect was minimized.

Some other limitations and future directions should also be mentioned. Firstly, the limited spatial accuracy of EEG should be borne in mind when interpreting the results. In this way the proposed correspondence between electrodes and cortical areas should be treated as putative but not certain. Secondly, the indirect inference about attentional characteristics was based on existing theoretical models that link the emotional state with changes in perception. Further verification of the results is recommended in future studies, where different task demands and attentional manipulation can be applied.

Acknowledgments The study was supported by a grant from the National Centre of Science (decision DEC-2011/01/D/HS6/00450).

Conflict of Interest Miroslaw Wyczesany, Tomasz S. Ligeza, and Szczepan J. Grzybowski declare that they have no conflicts of interest.

Informed Consent All procedures followed were in accordance with the ethical standards of the responsible committee on human experimentation (institutional and national) and with the Helsinki Declaration of 1975, and the applicable revisions at the time of the investigation. Informed consent was obtained from all patients for being included in the study. 
Open Access This article is distributed under the terms of the Creative Commons Attribution License which permits any use, distribution, and reproduction in any medium, provided the original author(s) and the source are credited.

\section{References}

Asplund, C. L., Todd, J. J., Snyder, A. P., \& Marois, R. (2010). A central role for the lateral prefrontal cortex in goal-directed and stimulusdriven attention. Nature Neuroscience, 13(4), 507-512. doi:10. 1038/nn.2509.

Benjamini, Y., \& Hochberg, Y. (1995). Controlling the false discovery rate: a practical and powerful approach to multiple testing. Journal of the Royal Statistical Society: Series B: Methodological, 57(1), 289-300.

Bishop, S. J. (2008). Neural mechanisms underlying selective attention to threat. Annals of the New York Academy of Sciences, 1129(1), 141152. doi:10.1196/annals.1417.016.

Bishop, S. J., Duncan, J., \& Lawrence, A. D. (2004). State anxiety modulation of the amygdala response to unattended threat-related stimuli. The Journal of Neuroscience, 24(46), 10364-10368. doi:10. 1523/JNEUROSCI.2550-04.2004.

Blinowska, K. J., Kuś, R., \& Kamiński, M. (2004). Granger causality and information flow in multivariate processes. Physical Review E, 70(5), 050902.

Büchel, C., \& Friston, K. J. (1997). Modulation of connectivity in visual pathways by attention: cortical interactions evaluated with structural equation modelling and fMRI. Cerebral Cortex, 7(8), 768-778. doi: 10.1093/cercor/7.8.768.

Buhle, J. T., Silvers, J. A., Wager, T. D., Lopez, R., Onyemekwu, C., Kober, H., \& Ochsner, K. N. (2013). Cognitive reappraisal of emotion: a meta-analysis of human neuroimaging studies. Cerebral Cortex (New York, N.Y.: 1991). doi:10.1093/cercor/ bht154.

Corbetta, M., \& Shulman, G. L. (2002). Control of goal-directed and stimulus-driven attention in the brain. Nature Reviews Neuroscience, 3(3), 201-215. doi:10.1038/nrn755.

Corbetta, M., Patel, G., \& Shulman, G. L. (2008). The reorienting system of the human brain: from environment to theory of mind. Neuron, 58(3), 306-324. doi:10.1016/j.neuron.2008.04.017.

D’Alfonso, A. A. L., van Honk, J., Hermans, E., Postma, A., \& de Haan, E. H. F. (2000). Laterality effects in selective attention to threat after repetitive transcranial magnetic stimulation at the prefrontal cortex in female subjects. Neuroscience Letters, 280(3), 195-198.

Dalton, K. M., Kalin, N. H., Grist, T. M., \& Davidson, R. J. (2005). Neural-cardiac coupling in threat-evoked anxiety. Journal of Cognitive Neuroscience, 17(6), 969-980. doi:10.1162/ 0898929054021094.

Delorme, A., Mullen, T., Kothe, C., Akalin Acar, Z., Bigdely-Shamlo, N., Vankov, A., \& Makeig, S. (2011). EEGLAB, SIFT, NFT, BCILAB, and ERICA: New tools for advanced EEG processing. Computational Intelligence and Neuroscience, 2011, 1-12. doi:10. 1155/2011/130714

Dijk, H., van Schoffelen, J. M., Oostenveld, R., \& Jensen, O. (2008). Prestimulus oscillatory activity in the alpha band predicts visual discrimination ability. The Journal of Neuroscience, 28(8), 18161823. doi:10.1523/JNEUROSCI.1853-07.2008.

Eysenck, M. W., Derakshan, N., Santos, R., \& Calvo, M. G. (2007). Anxiety and cognitive performance: attentional control theory. Emotion, 7(2), 336-353. doi:10.1037/1528-3542.7.2.336.

Fiedler, K., Nickel, S., Asbeck, J., \& Pagel, U. (2003). Mood and the generation effect. Cognition and Emotion, 17(4), 585-608. doi:10. 1080/02699930302301.
Flor, H., Knost, B., \& Birbaumer, N. (1997). Processing of pain-and body-related verbal material in chronic pain patients: central and peripheral correlates. Pain, 73(3), 413-421.

Fox, M. D., Corbetta, M., Snyder, A. Z., Vincent, J. L., \& Raichle, M. E. (2006). Spontaneous neuronal activity distinguishes human dorsal and ventral attention systems. Proceedings of the National Academy of Sciences, 103(26), 10046-10051.

Frank, D. W., \& Sabatinelli, D. (2012). Stimulus-driven reorienting in the ventral frontoparietal attention network: the role of emotional content. Frontiers in Human Neuroscience, 6, 116. doi:10.3389/fnhum. 2012.00116.

Gola, M., Magnuski, M., Szumska, I., \& Wróbel, A. (2013). EEG beta band activity is related to attention and attentional deficits in the visual performance of elderly subjects. International Journal of Psychophysiology, 89(3), 334-341. doi:10.1016/j.ijpsycho.2013. 05.007 .

Grzybowski, S., Wyczesany, M., \& Kaiser, J. (2014). The influence of context on the processing of emotional and neutral adjectives - an ERP study. Biological Psychology, 99, 137-149.

Gutiérrez, A., \& Gutiérrez Calvo, M. (2011). Foveal vs. Parafoveal processing in anxiety: broadened spatial attention for threat words. Psicológica: Revista de Metodología Y Psicología Experimental, 32(2), 301-321.

Gyurak, A., Gross, J. J., \& Etkin, A. (2011). Explicit and implicit emotion regulation: a dual-process framework. Cognition and Emotion, 25(3), 400-412. doi:10.1080/02699931.2010.544160.

Hedden, T., \& Gabrieli, J. D. E. (2006). The ebb and flow of attention in the human brain. Nature Neuroscience, 9(7), 863-865. doi:10.1038/ nn0706-863.

Herbert, C., Kissler, J., Junghöfer, M., Peyk, P., \& Rockstroh, B. (2006). Processing of emotional adjectives: evidence from startle EMG and ERPs. Psychophysiology, 43(2), 197-206.

Herbert, C., Junghofer, M., \& Kissler, J. (2008). Event related potentials to emotional adjectives during reading. Psychophysiology, 45(3), 487-498. doi:10.1111/j.1469-8986.2007.00638.x.

Hurlburt, R. T., \& Heavey, C. L. (2001). Telling what we know: describing inner experience. Trends in Cognitive Sciences, 5(9), 400-403. doi:10.1016/S1364-6613(00)01724-1.

Jung, T. P., Makeig, S., Humphries, C., Lee, T. W., Mckeown, M. J., Iragui, V., \& Sejnowski, T. J. (2000). Removing electroencephalographic artifacts by blind source separation. Psychophysiology, $37(02), 163-178$.

Kaiser, D. A. (2007). What is quantitative EEG? Journal of Neurotherapy, 10(4), 37-52.

Kamiński, M., \& Blinowska, K. (2014). Directed transfer function is not influenced by volume conduction - inexpedient pre-processing should be avoided. Frontiers in Computational Neuroscience, 8, 61 .

Kamiński, M., Ding, M., Truccolo, W. A., \& Bressler, S. L. (2001). Evaluating causal relations in neural systems: granger causality, directed transfer function and statistical assessment of significance. Biological Cybernetics, 85(2), 145-157.

Kamiński, J., Brzezicka, A., Gola, M., \& Wróbel, A. (2012). Beta band oscillations engagement in human alertness process. International Journal of Psychophysiology, 85(1), 125-128.

Kiefer, M., Schuch, S., Schenck, W., \& Fiedler, K. (2007). Emotion and memory: event-related potential indices predictive for subsequent successful memory depend on the emotional mood state. Advances in Cognitive Psychology, 3(3), 363-373.

Korzeniewska, A., Maczak, M., Kamiński, M., Blinowska, K. J., \& Kasicki, S. (2003). Determination of information flow direction among brain structures by a modified directed transfer function (dDTF) method. Journal of Neuroscience Methods, 125(1-2), 195-207.

Kotani, Y., Ohgami, Y., Kuramoto, Y., Tsukamoto, T., Inoue, Y., \& Aihara, Y. (2009). The role of the right anterior insular cortex in the right hemisphere preponderance of stimulus-preceding 
negativity (SPN): An fMRI study. Neuroscience Letters, 450(2), 7579. doi:10.1016/j.neulet.2008.11.032.

Kus, R., Blinowska, K. J., Kamiński, M., \& Basińska-Starzycka, A. (2008). Transmission of information during continuous attention test. Acta Neurobiologiae Experimentalis, 68(1), 103.

Lang, P. J., Bradley, M. M., \& Cuthbert, B. N. (1999). International affective picture system (IAPS): Instruction manual and affective ratings. The Center for Research in Psychophysiology, University of Florida.

Lee, K.-Y., Lee, T.-H., Yoon, S.-J., Cho, Y. S., Choi, J.-S., \& Kim, H. T. (2010). Neural correlates of top-down processing in emotion perception: an ERP study of emotional faces in white noise versus noise-alone stimuli. Brain Research, 1337, 56-63. doi:10.1016/j. brainres.2010.03.094.

Li, Y., Xiao, X., Ma, W., Jiang, J., Qiu, J., \& Zhang, Q. (2013). Electrophysiological evidence for emotional valence and competitive arousal effects on insight problem solving. Brain Research, 1538, 61-72. doi:10.1016/j.brainres.2013.09.021.

Lieberman, M. D., Eisenberger, N. I., Crockett, M. J., Tom, S. M., Pfeifer, J. H., \& Way, B. M. (2007). Putting feelings into words affect labeling disrupts amygdala activity in response to affective stimuli. Psychological Science, 18(5), 421-428. doi:10.1111/j.1467-9280. 2007.01916.x.

MacDonald, A. W., Cohen, J. D., Stenger, V. A., \& Carter, C. S. (2000). Dissociating the role of the dorsolateral prefrontal and anterior cingulate cortex in cognitive control. Science, 288(5472), 18351838. doi:10.1126/science.288.5472.1835.

MacLeod, C., \& Hagan, R. (1992). Individual differences in the selective processing of threatening information, and emotional responses to a stressful life event. Behaviour Research and Therapy, 30(2), 151161. doi:10.1016/0005-7967(92)90138-7.

Matthews, G., Jones, D. M., \& Chamberlain, A. G. (1990). Refining the measurement of mood: the UWIST mood adjective checklist. British Journal of Psychology, 81(1), 17-42.

McLaughlin, N. C. R., Moore, D. W., Fulwiler, C., Bhadelia, R., \& Gansler, D. A. (2009). Differential contributions of lateral prefrontal cortex regions to visual memory processes. Brain Imaging and Behavior, 3(2), 202-211. doi:10.1007/s11682-009-9062-7.

Michalowski, J. M., Melzig, C. A., Weike, A. I., Stockburger, J., Schupp, H. T., \& Hamm, A. O. (2009). Brain dynamics in spider-phobic individuals exposed to phobia-relevant and other emotional stimuli. Emotion, 9(3), 306

Min, B. K., \& Herrmann, C. S. (2007). Prestimulus EEG alpha activity reflects prestimulus top-down processing. Neuroscience Letters, 422(2), 131-135. doi:10.1016/j.neulet.2007.06.013.

Moratti, S., Saugar, C., \& Strange, B. A. (2011). Prefrontaloccipitoparietal coupling underlies late latency human neuronal responses to emotion. The Journal of Neuroscience, 31(47), 17278-17286. doi:10.1523/JNEUROSCI.2917-11.2011.

Niedenthal, P. M., \& Setterlund, M. B. (1994). Emotion congruence in perception. Personality and Social Psychology Bulletin, 20(4), 401411. doi:10.1177/0146167294204007.

Niedenthal, P. M., Jasmin, B. H., \& Setterlund, M. B. (1997). Being happy and seeing "Happy": emotional state mediates visual word recognition. Cognition and Emotion, 11(4), 403-432. doi:10.1080/ 026999397379863.

Nunez, P. L., Srinivasan, R., Westdorp, A. F., Wijesinghe, R. S., Tucker, D. M., Silberstein, R. B., \& Cadusch, P. J. (1997). EEG coherency: I: statistics, reference electrode, volume conduction, laplacians, cortical imaging, and interpretation at multiple scales. Electroencephalography and Clinical Neurophysiology, 103(5), 499-515.

Ochsner, K. N., Silvers, J. A., \& Buhle, J. T. (2012). Functional imaging studies of emotion regulation: a synthetic review and evolving model of the cognitive control of emotion. Annals of the New York Academy of Sciences, 1251, E1-24. doi:10.1111/j.1749-6632.2012.06751.x.
Okamoto, M., Dan, H., Sakamoto, K., Takeo, K., Shimizu, K., Kohno, S., \& Kohyama, K. (2004). Three-dimensional probabilistic anatomical cranio-cerebral correlation via the international 10-20 system oriented for transcranial functional brain mapping. NeuroImage, 21(1), 99-111.

Okon-Singer, H., Tzelgov, J., \& Henik, A. (2007). Distinguishing between automaticity and attention in the processing of emotionally significant stimuli. Emotion, 7(1), 147-157. doi:10.1037/15283542.7.1.147.

Oliveri, M., Zhaoping, L., Mangano, G. R., Turriziani, P., Smirni, D., \& Cipolotti, L. (2010). Facilitation of bottom-up feature detection following rTMS-interference of the right parietal cortex. Neuropsychologia, 48(4), 1003-1010. doi:10.1016/j. neuropsychologia.2009.11.024.

Romei, V., Gross, J., \& Thut, G. (2010). On the role of prestimulus alpha rhythms over occipito-parietal areas in visual input regulation: correlation or causation? The Journal of Neuroscience, 30(25), 86928697. doi:10.1523/JNEUROSCI.0160-10.2010.

Rossi, V., \& Pourtois, G. (2012). State-dependent attention modulation of human primary visual cortex: a high density ERP study. NeuroImage, 60(4), 2365-2378. doi:10.1016/j.neuroimage.2012.02.007.

Rowe, J. B., Stephan, K. E., Friston, K., Frackowiak, R. S. J., \& Passingham, R. E. (2005). The prefrontal cortex shows contextspecific changes in effective connectivity to motor or visual cortex during the selection of action or colour. Cerebral Cortex, 15(1), 8595. doi:10.1093/cercor/bhh111.

Rowe, G., Hirsh, J. B., \& Anderson, A. K. (2007). Positive affect increases the breadth of attentional selection. Proceedings of the National Academy of Sciences, 104(1), 383-388. doi:10.1073/pnas. 0605198104

Ruff, C. C., Bestmann, S., Blankenburg, F., Bjoertomt, O., Josephs, O., Weiskopf, N., \& Driver, J. (2008). Distinct causal influences of parietal versus frontal areas on human visual cortex: evidence from concurrent TMS-fMRI. Cerebral Cortex, 18(4), 817-827. doi:10. 1093/cercor/bhm128.

Salmi, J., Rinne, T., Koistinen, S., Salonen, O., \& Alho, K. (2009). Brain networks of bottom-up triggered and top-down controlled shifting of auditory attention. Brain Research, 1286, 155-164.

Serences, J. T., Shomstein, S., Leber, A. B., Golay, X., Egeth, H. E., \& Yantis, S. (2005). Coordination of voluntary and stimulus-driven attentional control in human cortex. Psychological Science, 16(2), 114-122. doi:10.1111/j.0956-7976.2005.00791.x.

Shackman, A. J., Maxwell, J. S., McMenamin, B. W., Greischar, L. L., \& Davidson, R. J. (2011). Stress potentiates early and attenuates late stages of visual processing. The Journal of Neuroscience, 31(3), 1156-1161. doi:10.1523/JNEUROSCI.3384-10.2011.

Silvers, J. A., Weber, J., Wager, T. D., \& Ochsner, K. N. (2014). Bad and worse: neural systems underlying reappraisal of high and low intensity negative emotions. Social Cognitive and Affective Neuroscience. doi:10.1093/scan/nsu043.

Stuss, D. T., Floden, D., Alexander, M. P., Levine, B., \& Katz, D. (2001). Stroop performance in focal lesion patients: dissociation of processes and frontal lobe lesion location. Neuropsychologia, 39(8), 771786. doi:10.1016/S0028-3932(01)00013-6.

Taylor, S. F., Phan, K. L., Decker, L. R., \& Liberzon, I. (2003). Subjective rating of emotionally salient stimuli modulates neural activity. NeuroImage, 18(3), 650-659. doi:10.1016/S1053-8119(02)00051-4.

Thomas, D. L., \& Diener, E. (1990). Memory accuracy in the recall of emotions. Journal of Personality and Social Psychology, 59(2), 291-297. doi:10.1037/0022-3514.59.2.291.

Todd, J. J., Fougnie, D., \& Marois, R. (2005). Visual short-term memory load suppresses temporo-parietal junction activity and induces inattentional blindness. Psychological Science, 16(12), 965-972. doi:10.1111/j.1467-9280.2005.01645.x.

Tukey, J. W. (1976). Exploratory data analysis. 1977. Massachusetts: Addison-Wesley. 
Tupak, S. V., Dresler, T., Guhn, A., Ehlis, A.-C., Fallgatter, A. J., Pauli, P., \& Herrmann, M. J. (2014). Implicit emotion regulation in the presence of threat: Neural and autonomic correlates. NeuroImage, 85, Part 1, 372-379. doi:10.1016/ j.neuroimage.2013.09.066

Vanderhasselt, M. A., Raedt, R. D., \& Baeken, C. (2009). Dorsolateral prefrontal cortex and stroop performance: tackling the lateralization. Psychonomic Bulletin and Review, 16(3), 609-612. doi:10.3758/ PBR.16.3.609.

Vanlessen, N., Rossi, V., Raedt, R. D., \& Pourtois, G. (2013). Positive emotion broadens attention focus through decreased position-specific spatial encoding in early visual cortex: evidence from ERPs. Cognitive, Affective, and Behavioral Neuroscience, 13(1), 60-79. doi:10.3758/ s13415-012-0130-x.

Vossel, S., Geng, J. J., \& Fink, G. R. (2013). Dorsal and ventral attention systems distinct neural circuits but collaborative roles. The Neuroscientist. doi:10.1177/1073858413494269.

Vuilleumier, P. (2005). How brains beware: neural mechanisms of emotional attention. Trends in Cognitive Sciences, 9(12), 585-594.

Wadlinger, H. A., \& Isaacowitz, D. M. (2006). Positive mood broadens visual attention to positive stimuli. Motivation and Emotion, 30(1), 87-99. doi:10.1007/s11031-006-9021-1.

Wen, X., Yao, L., Liu, Y., \& Ding, M. (2012). Causal interactions in attention networks predict behavioral performance. The Journal of
Neuroscience, 32(4), 1284-1292. doi:10.1523/JNEUROSCI.281711.2012.

Westermann, R., Spies, K., Stahl, G., \& Hesse, F. W. (1996). Relative effectiveness and validity of mood induction procedures: a metaanalysis. European Journal of Social Psychology, 26(4), 557-580. doi:10.1002/(SICI)1099-0992(199607)26:4<557::AIDEJSP769>3.0.CO;2-4.

Wyczesany, M., Kaiser, J., \& Coenen, A. (2008). Subjective mood estimation co-varies with spectral power EEG characteristics. Acta Neurobiologiae Experimentalis, 68, 180-192.

Wyczesany, M., Kaiser, J., \& Barry, R. (2009). Cortical lateralization patterns related to self-estimation of emotional state. Acta Neurobiologiae Experimentalis, 69(4), 526-536.

Wyczesany, M., Kaiser, J., \& Coenen, A. (2010). Associations between self-report of emotional state and the EEG patterns in affective disorders patients. Journal of Psychophysiology, $24,33-40$.

Wyczesany, M., Ferdek, M. A., \& Grzybowski, S. J. (2014). Cortical functional connectivity is associated with the valence of affective states. Brain and Cognition, 90, 109-115

Zanto, T. P., Rubens, M. T., Thangavel, A., \& Gazzaley, A. (2011). Causal role of the prefrontal cortex in top-down modulation of visual processing and working memory. Nature Neuroscience, 14(5), 656-661. doi:10.1038/nn. 2773. 